\title{
Application of a Reassortant Cucumber mosaic virus Vector for Gene Silencing in Tomato and Chili Pepper Plants
}

\author{
Jin Sung Hong ${ }^{1 \dagger}$, Sun-Ju Rhee ${ }^{2 \dagger}$, Eun-Ji Kim ${ }^{2}$, Tae-Sung Kim ${ }^{2}$, Ki Hyun Ryu ${ }^{1}$, Chikara Masuta ${ }^{3}$ and \\ Gung Pyo Lee ${ }^{2 *}$ \\ ${ }^{1}$ Plant Virus Genbank, Seoul Women's University, Seoul 139-774, Korea \\ ${ }^{2}$ Department of Integrative Plant Science, Chung-Ang University, Ansung 456-756, Korea \\ ${ }^{3}$ Research Faculty of Agriculture, Hokkaido University, Kita-ku, Sapporo, Japan \\ (Received on November 20, 2011; Revised on November 28, 2011; Accepted on November 28, 2011)
}

\begin{abstract}
We developed a reassortant RNA virus vector derived from Cucumber mosaic virus (CMV), which has advantages of very wide host range and can efficiently induce gene silencing in a few model plants. Certain CMV isolates, however, show limited host ranges presumably because they naturally co-evolved with their own hosts. We used a reassortant comprised of two strains of CMV, Y-CMV and Gn-CMV, to broaden the host range and to develop a virus vector for virus-induced gene silencing (VIGS). Gn-CMV could infect chili pepper and tomato more efficiently than Y-CMV. Gn-CMV RNA1, 3 and Y-CMV RNA2-A1 vector were newly reconstructed, and the transcript mixture of RNA1 and 3 genomes of Gn-CMV and RNA2 genome of Y-CMV RNA2 containing portions of the endogenous phytoene desaturase (PDS) gene (CMV2A1::PDSs) was inoculated onto chili pepper (cv. Chung-yang), tomato (cvs. Bloody butcher, Tigerella, Silvery fir tree, and Czech bush) and Nicotiana benthamiana. All the tested plants infected by the reassortant CMV vector showed typical photo-bleaching phenotypes and reduced expression levels of $P D S$ mRNA. These results suggest that the reassortant CMV vector would be a useful tool for the rapid induction of the RNA silencing of endogenous genes in chili pepper and tomato plants.
\end{abstract}

Keywords : Cucumber mosaic virus vector, endogenous gene, phytoene desaturase, RNA silencing

In plants, the RNA silencing mechanism is conserved and results from sequence-specific degradation of target RNAs, which has an essential role in most development stages. RNA silencing depends on small RNAs (21 to $24 \mathrm{nt}$ ), which are the key regulators of RNA silencing pathways in

\footnotetext{
${ }^{\dagger}$ Equally contributed.

*Corresponding author.

Phone) +82-31-670-3039, FAX) +82-31-676-6714

E-mail) gplee@cau.ac.kr
}

eukaryotic organisms (Fire et al., 1998; Pickford and Cogoni, 2003; Vance and Voucheret, 2001; Waterhouse et al., 2001). Recently the transcription of double-stranded RNA (dsRNA) was shown to effectively down-regulate a gene (Hamilton et al., 1998; Waterhouse et al., 1998). When viruse was manipulated to carry a region of the host gene sequences, the process can also be targeted against the corresponding mRNA. This is called virus-induced gene silencing (VIGS). After virus replication, replicative form (dsRNA) intermediates can trigger the RNA-mediated defense mechanism, and dsRNA intermediates are processed into short interfering RNAs (siRNAs). These small RNAs are produced from dsRNA by Dicer-like (DCL) enzymes, and act as guides in RNA-induced silencing complexes (RISCs) to target RNA or DNA sequences (Bernstein et al., 2001; Hamilton and Baulcombe, 1999; Hammond et al., 2000; Zamore et al., 2000). The phenotypic changes in the infected plant reflect the loss of expression of the encoded protein (Lu et al., 2003). Over the past 15 years, several RNA virus vectors have been developed for VIGS. These include Tobacco mosaic virus (Kumagai et al., 1995), Potato virus X (Ruiz et al., 1998), Tobacco rattle virus (Liu et al., 2002; Ratcliff et al., 1999), Barley stripe mosaic virus (Holzverg, 2002), Pea early browning virus (Constantin et al., 2004), Bean pod mottle virus (Zhang and Ghabrial, 2006), Cucumber mosaic virus (Nagamatsu, 2007; Otagaki et al., 2006), and Pepper mottle mosaic virus (Lee et al., 2011). VIGS has many advantages over other methods, in which it is rapid and does not require a recalcitrant plant transformation (Burch-smith et al., 2004). VIGS has been successfully applied to dicotyledonous species including Arabidopsis (Burch-Smith et al., 2006), potato (Brigneti et al., 2004), tomato (Liu et al., 2002), pepper (Chung et al., 2004) and to several monocots including barley (Holzberg et al., 2002; Hein et al., 2005), wheat (Scofield et al., 2005) and rice (Ding et al., 2006; Ding et al., 2007).

Cucumber mosaic virus (CMV) is one of the wellcharacterized positive-sense single stranded RNA viruses 
and has the widest host range including more than 1,200 species in over 100 families among the plant viruses (Edwardson and Christie 1991, Palukaitis et al., 1992). The CMV genome consists of the tripartite component RNAs (RNAs 1-3), and encodes five proteins (Palukaitis and Garcia-Arenal, 2003). RNA1 and RNA2 encode the 1a and 2a proteins, which are attributed viral replication (Hayes and Buck, 1990). RNA3 encodes the movement protein (3a) and coat protein (CP). CP is translated from subgenomic RNA4 (Palukaitis and Garcia-Arenal, 2003). Many studies have described that CMV host-range is determined by RNA3 of CMV (Carrere et al., 1999; Hong et al., 2007; Palukaitis and Garcia-Arenal, 2003). In addition, RNA2 encodes $2 b$ protein, which is known as a suppressor of RNA silencing and expressed from a second subgenomic RNA (RNA4a) (Ding et al., 1994; Guo and Ding, 2002). Recently, Otagaki et al. (2006) developed a novel CMV vector including Y-CMV RNA2-A1, which lacks the $\mathrm{C}$-terminal one-third of the intact $2 \mathrm{~b}$ protein as a consequence of introducing restriction sites for cloning a foreign fragment. They proposed that the modification of $2 \mathrm{~b}$ of Y-CMV RNA2 was more suitable for VIGS by minimizing suppression of RNA silencing. The phytoene desaturase (PDS) gene encodes an enzyme involved in carotenoid biosynthesis, and silencing of this gene results in photo-bleaching of plant leaves (Liu et al., 2002). The leaves turn white due to lack of carotenoids and destruction of chlorophyll by photo-oxidation (Kumigai et al., 1995). Here, we deliberately chose a newly isolated Gn-CMV (Rhee et al., 2011), which was shown to infect diverse crops including Solanaceae, Cucurbitaceae and Brassicaceae. We used the Gn-CMV strain to construct a reassortant CMV vector with Y-CMV RNA2-A1 and described the CMV vector system to induce silencing of endogenous $P D S$ in Solanaceous plants including chili pepper and tomato as well as Nicotiana benthamiana.

For this study, N. benthamiana, tomato (Solanum lycopersicum cv. Jo-myoung) and chili pepper (Capsicum annuит cv. Geum-top) were used as test plants. Seeds were sown and germinated in plug-trays supplemented with Woncho-mix potting mixture (Nong Kyeong Co., Jincheon, Korea). Two to 3-week old seedlings were transplanted to 9 $\mathrm{cm}$ diameter round pots, containing Woncho-mix, with one plant per pot. Growth room conditions were kept at $23 \pm 3^{\circ} \mathrm{C}$ and under $16 \mathrm{~h}$ extended day with $100 \mu \mathrm{E} \mathrm{sec}^{-1}$ $\mathrm{m}^{-2}$ light intensity with cool-fluorescent lamps.

We had already obtained full-length cDNA clones of the genomic RNAs 1-3 of Gn-CMV from our previous work (Rhee et al., 2011). The modified plasmid of CMV RNA2 (CMV2-A1) was developed as a viral vector by replacing the one-third of $2 \mathrm{~b}$ gene with a multiple cloning site containing restriction sites (Matsuo et al., 2007; Otagaki et al., 2006). Fragments of three orthologs of PDS including NbPDS, CaPDS, and SIPDS genes were amplified by RTPCR from $N$. benthamiana, C. annuum cv. Geum-top, and S. lycopersicum cv. Jo-myeong, respectively. The amplified fragments were treated with $S t u I$ and $M l u I$ and then inserted into pGEM-T vector, which was sequenced using the universal primers based on T7 and SP6 promoters in the vector. Analyzed sequences were searched from GenBank, and $P D S$ were compared with available data in NCBI database. Sequences of amplified NbPDS, CaPDS, and SIPDS fragment were exactly matched with previously registered accessions in GenBank (Accession No. DQ469932, X68058, and NM 001247166, respectively). Amplified length of each $P D S$ gene was $369 \mathrm{bp}(N b P D S), 505 \mathrm{bp}$ (CaPDS), and 408bp (SIPDS). Each of the amplified cDNA fragments of $N b P D S, C a P D S$, and SIPDS was cloned into StuI/MluI-cut CMV2-A1 clone, respectively (Fig. 1). Each of the ligated constructs was confirmed by digestion with restriction enzyme such as $S t u \mathrm{I}$ and $M l u \mathrm{I}$ (data not shown). Sequences of all the pCMV2-A1 derivatives were confirmed by sequencing at the SolGent Co. (Yusung, Korea). The reassortant CMV RNAs were in vitro transcribed from plasmids, the Gn-CMV RNA1 (Gn-CMV R1), CMV2A1::PDS and Gn-CMV RNA3 (Gn-CMV R3). Each clone of CMV2-A1 vector, Gn-CMV R1, and Gn-CMV R3 genome was linearized by NotI, SacI, and SacI, repectively,

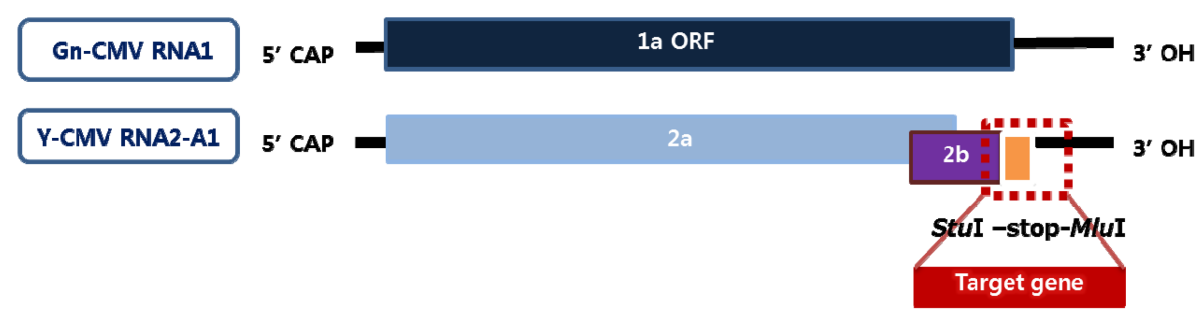

Gn-CMV RNA3

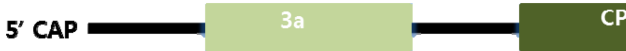

Fig. 1. Genome structure and construction of the reassortant CMV vector. RNAs 1-3 are the genomic RNAs of CMV. Each of the PDS genes were cloned between the Stu I and Mlu I sites of CMV2-A1 vector, followed by including Gn-CMV RNA1 and RNA3. 
prior to in vitro transcription using mMessage mMachine T7 kit (Ambion, Austin, TX, USA). To prepare inoculum, previously prepared 3 to $4 \mu \mathrm{g}$ of linearized DNA was transcribed in total $20 \mu \mathrm{l}$ reaction volume per each of the genome segment with the kit, including a 5' capping step of viral RNA with Cap analogue $\left(\mathrm{m}^{7} \mathrm{GpppG}\right)$ following the manufacturer's protocol. The newly developed two true leaves of 3-week-old plants were dusted with carborundum and rub-inoculated with $40 \mathrm{ul}$ of the inoculum, of which the mixed-volume ratio of Gn-CMV R1 : Y-CMV R2-A1 : GnCMV R3 was $2: 1: 1$. The inoculation method with in vitro transcripts was essentially the same for the three plant species. To confirm successful infection of virus in the plants without deletion of the inserted sequences was analysed by RT-PCR of the viral RNA. Later, tomato and chili pepper plants were inoculated from the sap of $N$. benthamiana leaves shown PDS gene silencing, as VIGS symptoms were not obtained by direct transcription inoculation.

The leaf-bleaching phenotype of $P D S$ silencing in leaves by CMV VIGS vectors was observed with different degrees at $N$. benthamiana, chili pepper, and tomato plants with the sequence-homology dependent tendency. CMV including CMV2-A1 containing the NbPDS, CaPDS, and SlPDS, respectively, was inoculated to $N$. benthamiana (Fig. 2). Prior to the inoculation to the cotyledons of tomato and chili pepper plants, CMV2-A1::PDSs were inoculated to $N$. benthamiana, of which leaves shown white phenotype were harvested and used as the inoculum for the above two crops. When direct transcript was inoculated to tomato and pepper, $P D S$ insert and CP of CMV were detected with low level in the upper leaves, although sometimes those plants showed partial photo-bleaching phenotype. We supposed that the amount of CMV replication could not reach the titer for VIGS by direct transcript inoculation in tomato and pepper. Although VIGS symptoms were not directly obtained from the inoculation of in vitro transcripts of CMV VIGS vectors, subsequent inoculation of sap obtained from $N$. benthamiana was successful to observe the white photo-bleaching phenotype in all of two crops within 20 dpi (Fig. 2). The silencing photo-bleached white phenotype, started to appear in the upper leaves with mild typical
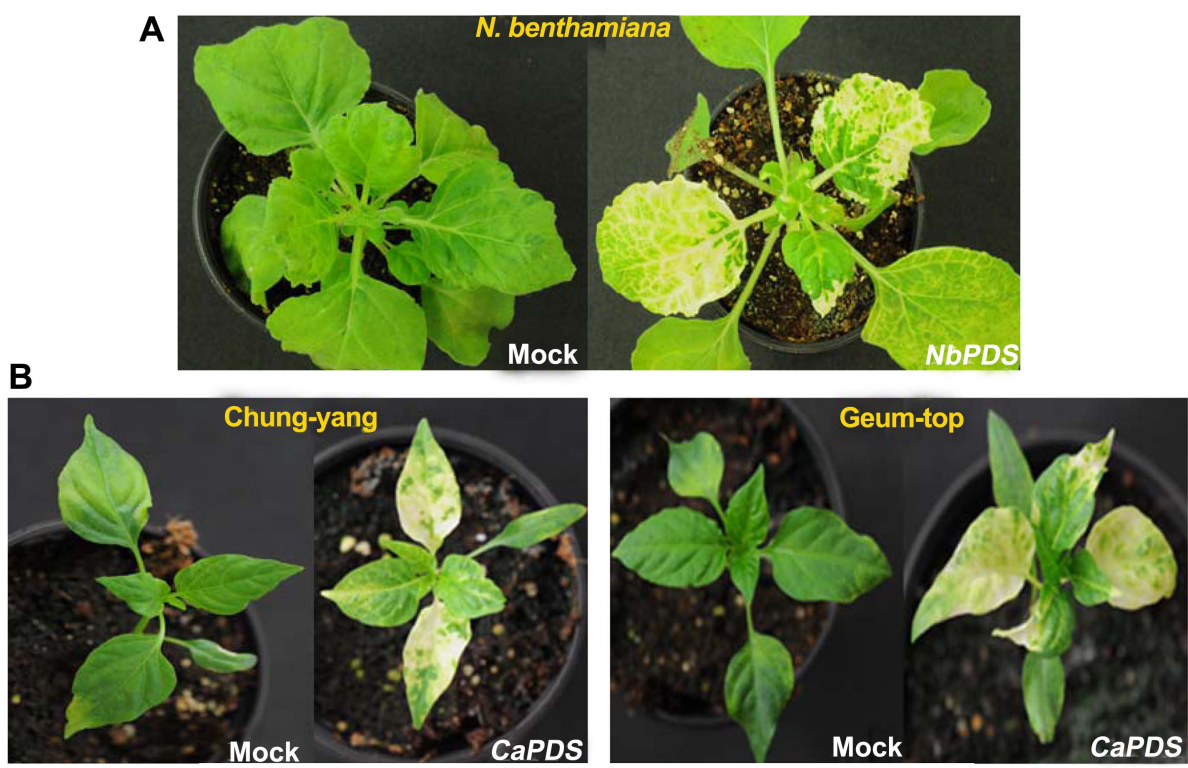

C
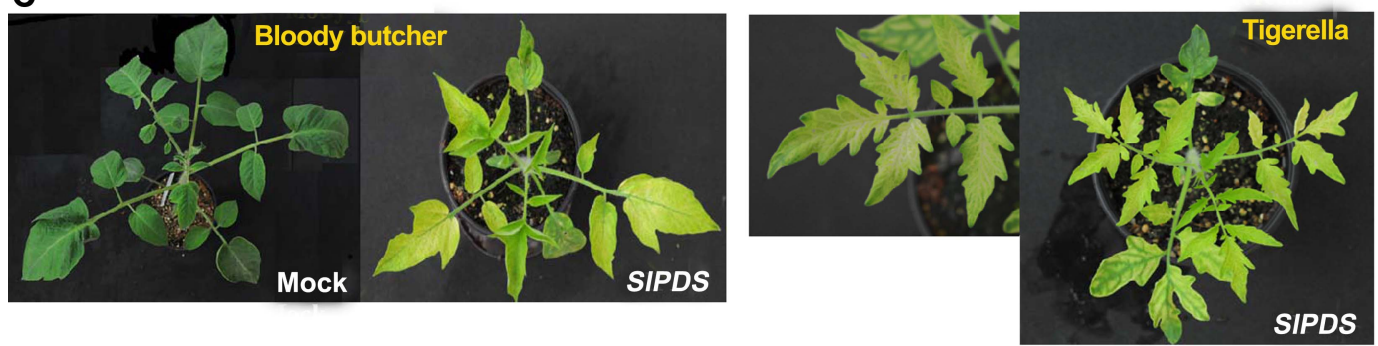

Fig. 2. Virus-induced gene silencing of endogenous $P D S$ gene showed photobleaching phenotype in three host plants by Gn-CMV containing CMV2-A1::PDS. (A) Infection of CMV2-A1::00 (empty vector, mock) and CMV2-A1::NbPDS in N. benthamiana at $11 \mathrm{dpi}$. (B) Infection of CMV2-A1::CaPDS in C. annuum cv. Chung-yang (left) and cv. Geum-top (right) at 12 dpi. (C) S. lycopersicum cv. Bloody butcher and cv. Tigerella infected with CMV2-A1::SIPDS 19 dpi. 
Table 1. List of primers used to amplification of $P D S$ genes for cloning into CMV2-A1 vector

\begin{tabular}{llcc}
\hline \hline Primer & Sequence & Enzyme & Product size \\
\hline NbPDS-F & 5'-CGGAGGCCTCTGACGAGCTTTCGATGCAG-3' & Stu I & $369 \mathrm{bp}$ \\
NbPDS-R & 5'-CGGACGCGTATATATGGATTTATCACA-3' & Mlu I & Stu I \\
CaPDS-F & 5'-CGGAGGCCTTGCTGTCAACTTGAGAGTCCA-3' & $505 \mathrm{bp}$ \\
CaPDS-R & 5'-CGGACGCGTGGTTCTGCATATTTGGGTAA-3' & Mlu I & $408 \mathrm{bp}$ \\
SIPDS-F & 5'-CGGAGGCCTGGCACTCAACTTTATAAA-3' & Stu I & \\
SIPDS-R & 5'-CGGACGCGTCTTCAGTTTCTGTCAAACC-3' & Mlu I & \\
\hline
\end{tabular}

Underline represent additional sequences to the target mRNA for generating restriction enzyme sites, Stu I and Mlu I.

mosaic symptom of CMV from 5 day post inoculation (dpi). This is in agreement with previous reports of CMV infection of $N$. tabacum and $N$. benthamiana and observed mosaic symptom (Canto et al., 1997) and the typical photobleaching phenotype induced by the silencing of $P D S$ gene using Tobacco rattle virus (TRV) in $N$. benthamiana (Liu et al., 2002; Liu and Page, 2008). PDS can be a good marker and target for endogenous gene silencing (Ruiz et al., 1998) in plants, because silenced plants show easily ascertainable and representative photo-bleached white phenotype, caused by the interruption of the carotenoid biosynthesis pathway.

Previously developed VIGS system have been successfully used for ascertaining the function of endogenous genes and applied to functional genomics in plants, but those virus vectors showed limited host ranges like Solanum species such as tobacco, tomato, and pepper plants. As a result of various recent research, several groups have reported successful VIGS in various plants such as cassava (Fofana et al., 2004), barley (Bruun-Rasmussen et al., 2007; Naylor et al., 2005), soybean (Nagamatsu et al., 2007), medicago and lathyrus (Gronlund et al., 2008). Recently, we have developed a vector for foreign gene cloning by manipulating RNA2 of Gn-CMV, which has a wide range of host plants and shows higher infectivity than Y-CMV in tomato and chili pepper, however, the virus vector composed of only Gn-CMV genomes showed VIGS symptoms with very low efficiency even in $N$. benthamiana (data not shown). One advantage of adapting the CMV vector is its wide host range. Different strains of CMV can infect different plants species and those pseudo-recombinants and recombinants can alter host ranges by changing RNA 3 genome (Carrere et al., 1999). Thus, reassortant CMVs would be an alternative approach to enhance VIGS in plants.

To confirm that the observed phenotypic changes in leaf photo-bleaching involve VIGS of the PDS genes, up- and down-regulations of the mRNA levels of $P D S$ genes after infection of the reassortant CMV vector were analyzed by semi-quantitative RT-PCR of total RNA extracted from the leaf of $N$. benthamiana, pepper and tomato plants. Total RNA was extracted from $0.4 \mathrm{~g}$ of $P D S$-gene silenced leaves of test plants using TRIZOL reagent (GIBCO-BRL-Life technologies, Gaithersburg, MD, USA) for cDNA synthesis. Actin genes of all test plants were amplified as the internal control using actin specific primer set (forward, 5'-GAAGATACTCACAGAAAGAGG-3'; reverse, 5'-GGAGCTAATGCAGTAA-TTTCC-3'). In $P D S$ gene silenced plants, expression levels of $P D S$ mRNA were examined by semiquantitative RT-PCR using each of their endogenous $P D S$ specific primer set (NbPDS1-F: 5'-CTCACGCCCAACTAAACCAT-3', NbPDS1-R: 5'-ATGTTCCTTCCACTGCAACC-3', CaPDS1-F: 5'-AATTTGCTCTTCAGCAGAAGCCCAC-3', CaPDS1-R: 5'-CTCTTGCAAAGATCCCCTGTAGAGGG-3', SIPDS1-F: 5'-TTGGGGCCTTTAAAGGTCGTATGC-3', SIPDS1-R: 5'-AGGAGCGGGTAAAGCTTCGGAGAA-3'). The First-strand cDNA was used as a template for PCR amplification through 18, 21, 24, 27, and 30 cycles. PCR reaction condition was as follows. Five minutes of initial denaturation followed by 35 cycles of $95^{\circ} \mathrm{C}$ for $30 \mathrm{sec}, 60^{\circ} \mathrm{C}$ (PDSs) and $58^{\circ} \mathrm{C}$ (Actin) for $1 \mathrm{~min}$ and $72^{\circ} \mathrm{C}$ for $1.5 \mathrm{~min}$, and finished with final elongation at $72{ }^{\circ} \mathrm{C}$ for $10 \mathrm{~min}$. The amplified products were separated on $1 \%$ agarose gel.

The mRNA levels of the PDS genes in $N$. benthamiana, chili pepper, and tomato leaves were significantly decreased by infection with reassortant CMV2-A1 containing $N b P D S$ (Fig. 3A), CaPDS (Fig. 3B), and SIPDS (Fig. 3C, $D)$. Quantification of the signal intensity of semi-quantitative RT-PCR comparing to actin gene as an internal control indicated that the PDS mRNA levels were reduced compared to the levels in control plants and the empty vector infected plants at 27 to 30 cycles (Fig. 3). These results indicate that the photo-bleaching phenotypic changes in leaf of CMV2-A1::PDSs infected plants were caused by VIGS of their endogenous $P D S$ genes.

To our knowledge, there has been no report relating to the application of a CMV vector silencing endogenous genes of chili pepper, although TRV vector has been successfully applied to chili pepper (Chung et al., 2004) and tomato (Burch-Smith et al., 2004) for VIGS. To study multiple gene function and virus-virus interactions in plants, development of more effective virus vectors is required. Comparing to other virus vectors, CMV vector would be easily broad- 

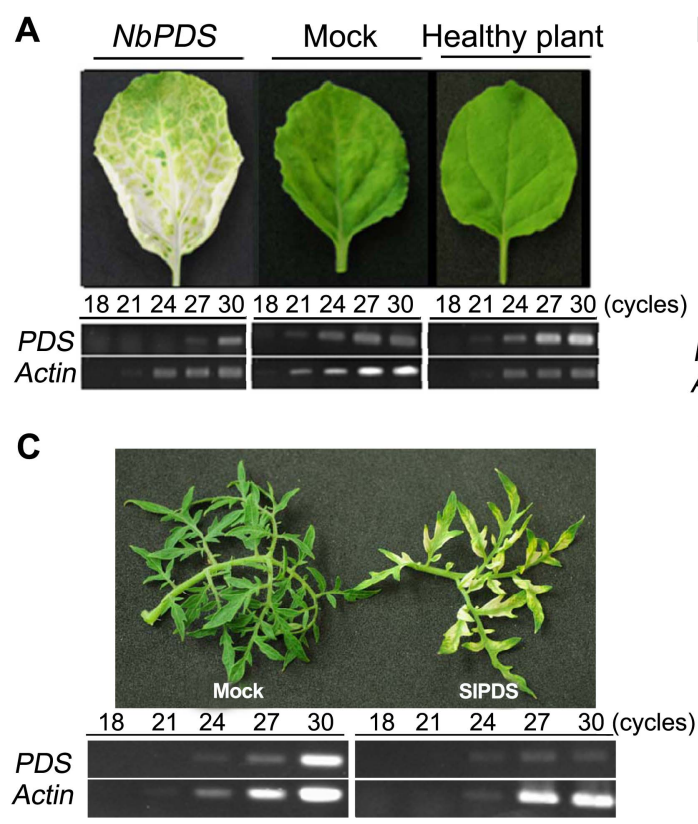
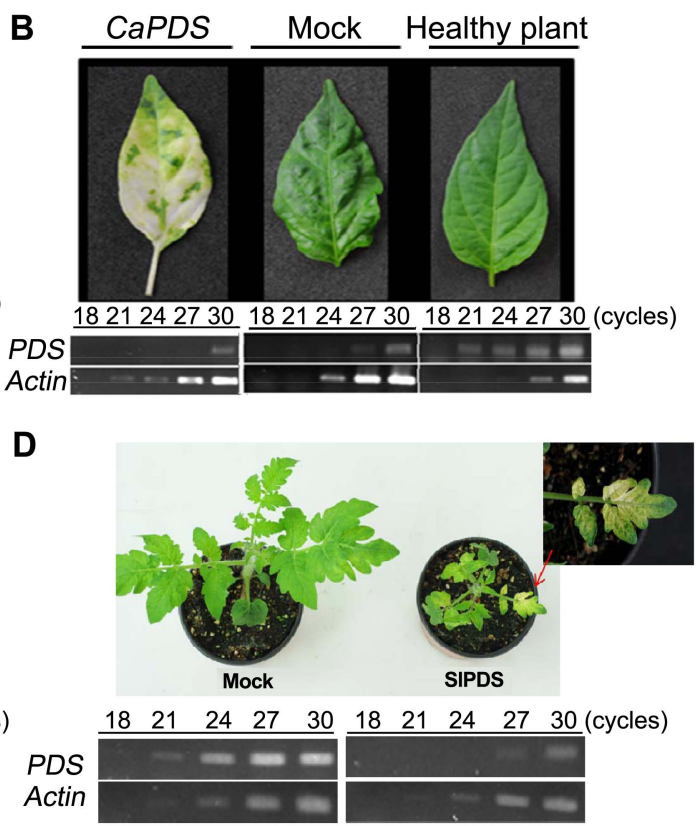

Fig. 3. Semi-quantitative RT-PCR in $N$. benthamiana, chili pepper, and two cultivars of tomato. (A) N. benthamiana infected with CMV2-A1::NbPDS at 15 dpi. (B) C. annuum cv. Chung-yang infected with CMV2-A1::CaPDS at 20 dpi. (C) S. lycopersicum cv. Silvery fir tree infected with CMV2-A1::SIPDS at 40 dpi. (D) S. lycopersicum cv. Czech bush infected with CMV2-A1::SIPDS at 19 dpi. Mock is the plant infected with CMV2-A1::00. Confirmation of PDS mRNA levels were performed using 18, 21, 24,27 and 30 cycles of RTPCR with tomato $P D S$ specific primer. The same actin-specific primer was used as control RT-PCR in all test plants.

ened its host ranges by changing RNA3 genome of other strains (Hong et al., 2007). In this study, we constructed a reassortant CMV vector and successfully showed PDS silencing in $N$. benthamiana, a chili pepper cv. Chung-yang and four cultivars of tomato including cvs. Bloody butcher, Tigerella, Silvery fir tree, and Czech bush. We expect that this CMV vector would be able to meet the needs to screen and to ascertain certain gene functions in chili pepper and tomato plants.

\section{Acknowledgements}

This research was supported by the Chung-Ang University Research Scholarship Grants in 2010.

\section{References}

Brigneti, G., Martin-Hernandez, A. M., Jin, H., Chen, J., Baulcombe, D. C., Baker, B. and Jones, J. D. 2004. Virus-induced gene silencing in Solanum species. Plant J. 39:264-272.

Bernstein, E., Caudy, A. A., Hammond, S. M. and Hannon, G. J. 2001. Role for a didentate ribonuclease in the initiation step of RNA interference. Nature 409:363-366.

Bruun-Rasmussen, M., Madsen, C. T., Jessing, S. and Albrechtsen, M. 2007. Stability of Barley stripe mosaic virus-induced gene silencing in barley. Mol. Plant-Microbe Interact. 20: $1323-1331$.

Burch-Smith, T. M., Anderson, J. C., Martin, G. B. and Dinesh-
Kumar, S. P. 2004. Applications and advantages of virusinduced gene silencing for gene function studies in plants. Plant J. 39:734-746.

Burch-Smith, T. M., Schiff, M., Liu, Y. and Dinesh-Kumar, S. P. 2006. Efficient virus-induced gene silencing in Arabidopsis. Plant Physiol. 142:21-27.

Canto, T., Prior, D. A., Hellwald, K. H., Oparka, K. J. and Palukaitis, P. 1997. Characterization of Cucumber mosaic virus. IV. Movement protein and coat protein are both essential for cell-to-cell movement of Cucumber mosaic virus. Virology 237:237-248.

Carrere, I., Tepfer, M. and Jacquemond, M. 1999. Recombinants of Cucumber mosaic virus (CMV): determinants of host range and symptomatology. Arch. Virol. 144:365-379.

Chung, E., Seong, E., Kim, Y. C., Chung, E. J., Oh, S. K., Lee, S., Park, J. M., Joung, Y. H. and Choi, D. 2004. A method of high frequency virus-induced gene silencing in chili pepper (Capsicum annuum L. cv. Bukang). Mol. Cells 17:377-380.

Constantin, G. D., Krath, B. N., MacFarlane, S. A., Nicolaisen, M., Johansen, I. E. and Lund, O. S. 2004. Virus-induced gene silencing as a tool for functional genomics in a legume species. Plant J. 40:622-631.

Ding, S. W., Anderson, B. J., Haase, H. R. and Symons, R. H. 1994. New overlapping gene encoded by the Cucumber mosaic virus genome. Virology 198:593-601.

Ding, X. S., Rao, C. S. and Nelson, R. S. 2007. Analysis of gene function in rice through virus-induced gene silencing. Methods Mol. Biol. 354:145-160.

Ding, X. S., Schneider, W. L., Chaluvadi, S. R., Mian, M. A. and 
Nelson, R. S. 2006. Characterization of a Brome mosaic virus strain and its use as a vector for gene silencing in monocotyledonous hosts. Mol. Plant-Microbe Interact. 19:1229-1239.

Edwardson, J. R. and Christie, R. G. 1991. Cucumoviruses. In; CRC Handbook of viruses infecting legumes, CRC Press, Boca Raton, pp. 293-319.

Fire, A., Xu, S. Montgomery, M. K., Kostas, S. A., Driver, S. E. and Mello, C. C. C. 1998. Potent and specific genetic interference by double-stranded RNA in Caenorhabditis elegans. Nature 391:806-811.

Fofana, I. B. F., Sangar, A., Collier, R., Taylor, C. and Fauquet, C. M. 2004. A geminivirus-induced gene silencing system for gene function validation in cassava. Plant Mol. Biol. 56:613-624.

Gronlund, M., Constantin, G., Piednoir, E., Kovacev, J., Johansen, I. E. and Lund, O. S. 2008. Virus-induced gene silencing in Medicago truncatula and Lathyrus odorata. Virus Res. 135: 345-349.

Hamilton, A. J. and Baulcombe, D. C. 1999. A species of small antisense RNA in posttranscriptional gene silencing in plants. Science 286:950-952.

Hammond, S. M., Bernstein, E., Beachy, D. and Hannon, G. J. 2000. An RNA-directed nuclease mediates post-transcriptional gene silencing in Drosophila cells. Nature 404:293-296.

Hayes, R. J. and Buck, K. W. 1990. Infectious Cucumber mosaic virus RNA transcribed in vitro from clones obtained from cDNA amplified using the polymerase chain reaction. J. Gen. Virol. 71:2503-2508.

Hein, I., Barciszewska-Pacak, M., Hrubikova, K., Williamson, S., Dinesen, M., Soenderby, I. E., Sundar, S., Jarmolowski, A., Shirasu, K. and Lacomme, C. 2005. Virus-induced gene silencing-based functional characterization of genes associated with powdery mildew resistance in barley. Plant Physiol. 138:2155-2164.

Holzberg, S., Brosio, P., Gross, C. and Pogue, G. P. 2002. Barley stripe mosaic virus-induced gene silencing in a monocot plant. Plant J. 30:315-327.

Hong, J. S., Ohnishi, S., Masuta, C., Choi, J. K. and Ryu, K. H. 2007. Infection of soybean by Cucumber mosaic virus as determined by viral movement protein. Arch. Virol. 152:321-328.

Kumagai, M. H., Donson, J., Della-Cioppa, G., Harvey, D., Hanley, K. and Grill, L. K. 1995. Cytoplasmic inhibition of carotenoid biosynthesis with virus-derived RNA. Proc. Natl. Acad. Sci. USA 92:1679-1683.

Lee, M. Y., Song, Y. S. and Ryu, K. H. 2011. Development of infectious transcripts from full-length and GFP-tagged cDNA clones of Pepper mottle virus and stable systemic expression of GFP in tobacco and pepper. Virus Res. 155:487-494.

Liu, E. and Page, J. E. 2008. Optimized cDNA libraries for virusinduced gene silencing (VIGS) using Tobacco rattle virus. Plant Methods 4:5.

Liu, Y., Schiff, M. and Dinesh-Kumar, S. P. 2002. Virus-induced gene silencing in tomato. Plant J.31:777-786.

Lu, R. Martin-Hernandez, A. M., Peart, J. R., Malcuit, I. and Baulcombe, D. C. 2003. Virus-induced gene silencing in plants. Methods 30:296-303.

Liu, Y., Schiff, M., Marathe, R. and Dinesh-Kumar, S. P. 2002.
Tobacco Rar1, EDS1 and NPR1/NIM1 like genes are required for N-mediated resistance to Tobacco mosaic virus. Plant $J$. 30:415-429.

Matsuo, K., Hong, J. S., Tabayashi, N., Ito, A., Masuta, C. and Matsumura, T. 2007 Development of Cucumber mosaic virus as a vector modifiable for different host species to produce therapeutic proteins. Planta 225:277-286.

Nagamatsu, A., Masuta, C., Senda, M., Matsuura, H., Kasai, A., Hong, J. S., Kitamura, K., Abe, J. and Kanazawa, A. 2007. Functional analysis of soybean genes involved in flavonoid biosynthesis by virus-induced gene silencing. Plant Biotechnol. J. 5:778-790.

Naylor, M., Reeves, J., Cooper, J. I., Edwards, M. L. and Wang, H. 2005. Construction and properties of a gene-silencing vector based on Poplar mosaic virus (genus Carlavirus). J. Virol. Methods 124:27-36.

Otagaki, S., Arai, M., Takahashi, A., Goto, K., Hong, J. S., Masuta, C. and Kanazawa, A. 2006. Rapid induction of transcriptional and post-transcriptional gene silencing using a novel Cucumber mosaic virus vector. Plant Biotechnol. 23:259-265.

Palukaitis, P., Garcia-Arenal, F. 2003. Cucumoviruses. Adv. Virus Res. 62:241-323.

Palukaitis, P., Roossinck, M. J., Dietzgen, R. G. and Francki, R. I. 1992. Cucumber mosaic virus. Adv. Virus Res. 41:281-348.

Pickford A. S. and Cogni, C. 2003. RNA mediated gene silencing. Cell. Mol. Life Sci. 60:871-882.

Ratcliff, F. G., Harrison, B. D. and Baulcombe, D. C. 1997. A similarity between viral defense and gene silencing in plants. Science 276:1558-1560.

Rhee, S. J., Hong, J. S., Choi, J. K., Kim, E. J. and Lee, G. P. 2011. Characterization of an isolate of Cucumber mosaic virus from Raphanus sativus L. Res. Plant Dis. 17:211-215.

Ruiz, M. T., Voinnet, O. and Baulcombe, D. C. 1998. Initiation and maintenance of virus-induced gene silencing. Plant Cell 10:937-946.

Scofield, S. R., Huang, L., Brandt, A. S. and Gill, B. S. 2005. Development of a virus-induced gene-silencing system for hexaploid wheat and its use in functional analysis of the Lr21mediated leaf rust resistance pathway. Plant Physiol. 138: 2165-2173.

Vance, V. and Vaucheret, H. 2001. RNA silencing in plantdefense and counter-defense. Science 292:2277-2280.

Waterhouse, P., Graham, M. and Wang, M. 1998. Virus resistance and gene silencing in plants can be induced by simultaneous expression of sense and antisense RNA. Proc. Natl. Acad. Sci. USA 95:13959-13964.

Waterhouse, P. M., Wang, M. B. and Lough, T. 2001. Gene silencing as an adaptive defense against viruses. Nature 411:834-842.

Zamore, P. D., Tuscho, T., Sharp, P. A. and Bartel, D. P. 2000. RNAi: double-stranded RNA directs the ATP-dependent cleavage of mRNA at 21 to 23 nucleotide intervals. Cell 101:25-33.

Zhang, C. Q. and Ghabrial, S. A. 2006. Development of Bean pod mottle virus-based vectors for stable protein expression and sequence-specific virus-induced gene silencing in soybean. Virology 344:401-411. 